\title{
Cardioprotective effects of autophagy induction in sepsis
}

\author{
Mahmoud Abdellatif ${ }^{1}$, Simon Sedej, ${ }^{1,2}$, Frank Madeo $^{2,3}$, Guido Kroemer $^{4,5,6,7,8,9,10}$ \\ ${ }^{1}$ Department of Cardiology, Medical University of Graz, Graz, Austria; ${ }^{2}$ BioTechMed Graz, Graz, Austria; ${ }^{3}$ Institute of Molecular Biosciences, \\ NAWI Graz, University of Graz, Graz, Austria; ${ }^{4}$ Equipe 11 Labellisée Ligue Contre le Cancer, Centre de Recherche des Cordeliers, Paris, France; \\ ${ }^{5}$ Cell Biology and Metabolomics Platforms, Gustave Roussy Comprehensive Cancer Center, Villejuif, France; ${ }^{6}$ INSERM, U1138, Paris, France; \\ ${ }^{7}$ Université Paris Descartes, Sorbonne Paris Cité, Paris, France; ${ }^{8}$ Université Pierre et Marie Curie, Paris, France; ${ }^{9}$ Pôle de Biologie, Hôpital \\ Européen Georges Pompidou, Paris, France; ${ }^{10}$ Karolinska Institute, Department of Women's and Children's Health, Karolinska University Hospital, \\ Stockholm, Sweden \\ Correspondence to: Frank Madeo. Institute of Molecular Biosciences, NAWI Graz, University of Graz, Graz, Austria. Email: frank.madeo@uni-graz.at; \\ Guido Kroemer. Equipe 11 Labellisée Ligue Contre le Cancer, Centre de Recherche des Cordeliers, Paris, France. Email: Kroemer@orange.fr. \\ Provenance: This is an invited Editorial commissioned by Section Editor Wenjian Jiang, MD (Department of Cardiac Surgery, Beijing Anzhen \\ Hospital, Capital Medical University, Beijing, China; Beijing Lab for Cardiovascular Precision Medicine, Beijing, China). \\ Comment on: Sun Y, Yao X, Zhang QJ, et al. Beclin-1-dependent autophagy protects the heart during sepsis. Circulation 2018. [Epub ahead of print].
}

Submitted Sep 26, 2018. Accepted for publication Oct 11, 2018.

doi: $10.21037 /$ atm.2018.10.23

View this article at: http://dx.doi.org/10.21037/atm.2018.10.23

\section{Introduction}

Macroautophagy (herein referred to as autophagy) is a protein and organelle quality control process that is essential for homeostasis and survival of many if not all eukaryotic cell types, including those composing the cardiovascular system. During autophagy, long-lived, dysfunctional and damaged cytoplasmic constituents are safely degraded into molecular building blocks for anabolic cellular renewal, energy production or detoxification (1). The process begins with the sequestration of such potentially toxic cellular material—designated for degradation-in de novo formed double-membraned vesicles, called autophagosomes. These then fuse with lysosomes to form autolysosomes, where their cargo undergoes enzymatic degradation by lysosomal acidic hydrolases (1). Remarkably, autophagy can operate in a selective and non-selective manner (2). Selective forms of autophagy are initiated to remove specific organelles or molecules, which typically pose harm to the cell. A wellcharacterized example of a highly specialized form of autophagy is mitophagy, where damaged and dysfunctional mitochondria are removed to minimize oxidative stress and protect from cell death. In contrast, non-selective autophagy is activated during times of nutrient deficit to maintain energy levels sufficient for sustaining cellular metabolism and functionality. Via providing versatile homeostatic resolution, autophagy plays a fundamental role in the maintenance of cellular fitness and overall organismal homeostasis under physiological and stress conditions, including starvation, hypoxia, physical exercise and immune responses to pathogens (1). As such, autophagy relies on a complex and well-orchestrated machinery and a wide regulatory network (3).

Beclin 1, the mammalian orthologue of yeast autophagyrelated protein (ATG)-6, is a key regulator within this autophagic cascade. Beclin 1 regulates different phases of autophagy through its interaction with PI3 KC3/ VPS34 (class III phosphatidylinositol 3-kinase/vacuolar protein sorting-34) lipid kinase. After the formation of the Beclin 1-PI3K3/VPS34 complex, other co-factors interact with Beclin 1 to control its autophagic activities (4). Specifically, ATG14 binds heterodimerically with Beclin 1, thereby forming the Beclin 1-PI3KC3/VPS34-ATG14 complex to promote autophagosome nucleation (an early stage of autophagosome formation). Another co-factor is UVRAG (UV radiation resistance-associated gene), which upon binding to Beclin 1-PI3KC3/VPS34 complex stimulates a later phase of autophagy, namely autolysosomal maturation. In contrast, Beclin 1 is blocked by anti-apoptotic multidomain proteins from the BCL2 family, which interact with the BCL2 homology 3 (BH3) domain of Beclin 1, thereby preventing its pro-autophagic activation by ATG14 or UVRAG (4). Beclin 1 is not only implicated in autophagy 


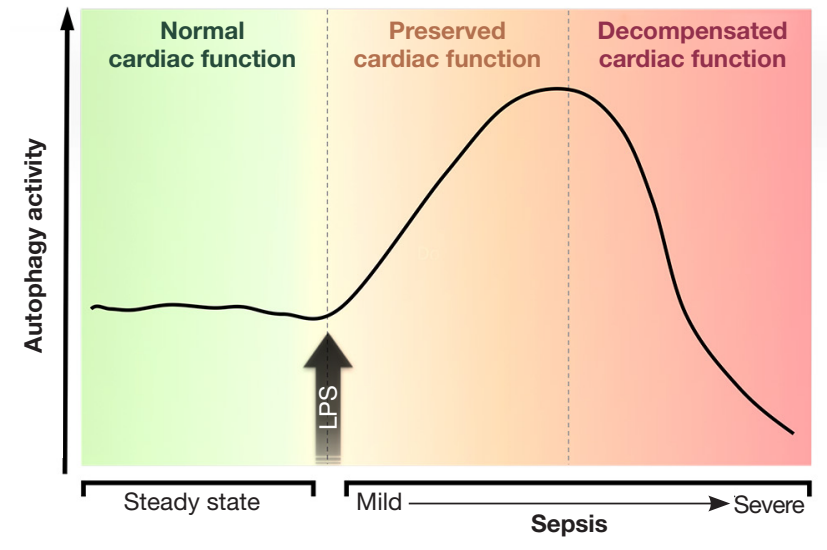

Figure 1 Bi-phasic modulation of autophagy during sepsis. During mild sepsis induced by low doses of LPS, autophagy appears to be activated in a dose-dependent manner, an effect that is associated with preserved cardiac function. In contrast, high doses of LPS, modelling severe sepsis, disable autophagy and induce cardiac dysfunction. LPS, lipopolysaccharide.

but also in other membrane trafficking processes, such as endocytosis (4). Accordingly, Beclin 1 is a pivotal modulator of vitally important biological processes. In fact, homozygous deletion of Beclin 1 in mice results in early lethality during embryogenesis, while its heterozygous disruption promotes premature tumorigenesis (5). Contrarily, activation of Beclin 1 (via a knock-in mutation that decreases BCL2 inhibitory binding) promotes basal autophagy and improves different aspects of health during the course of aging, including unwanted cardiac, renal and oncogenic alterations, thus extending the lifespan of mice (6).

\section{Beclin 1-mediated autophagy is cardioprotective in sepsis}

Sepsis is a leading cause of intensive care unit admission and subsequent mortality likely due to a dysregulated systemic inflammatory response that typically results from a serious infection (7). If not adequately and timely controlled by antibiotics, sepsis leads to a precipitous reduction in blood pressure and multiple organ failure, a life-threatening condition termed septic shock (8). Although the primary cause of death in these critically-ill patients is still enigmatic (7), a key determinant of outcome is the cardiovascular system performance (9). Interestingly, unlike other forms of shock, compromised cardiac output is not a typical characteristic of septic shock, albeit myocardial depression can be present in the form of reduced ejection fraction and dilation of both ventricles (8). Instead, the major hemodynamic crisis during septic shock is a severe drop in systemic vascular resistance, which leads to increased fluid permeability and plasma leakage to the extravascular compartments, resulting in 'distributive shock' (8).

Various clinical trials testing anti-inflammatory drugs in sepsis failed to improve patients' prognosis, thus, shedding doubts on the hypothesis that a fulminant immune reaction is the only culprit in sepsis-induced organ dysfunction (7). Therefore, other mechanisms that may contribute to the pathogenesis of sepsis are being extensively explored with the aim of providing novel therapeutic avenues for efficiently attenuating or reversing the associated organ dysfunction. In this regard, Sun et al. (10) carried out a cogent study that assessed autophagy in an animal model of sepsis induced by lipopolysaccharide (LPS), while focusing on cardiac parameters. In line with a previous liver-centric report (11), they observed inhibition of cardiac autophagy during severe sepsis, but more importantly, they uncovered a bi-phasic modulation of autophagy according to the severity of sepsis (Figure 1). On the one hand, during mild or early-stage sepsis imitated by low LPS doses, cardiac autophagy is increased in a dose-dependent manner, coinciding with preserved cardiac function. In contrast, autophagy is progressively disabled and cardiac function is clearly impaired upon administration of higher LPS doses, which induce a pathology resembling severe or late stages of sepsis (10).

To examine whether such autophagy modulation in the heart underlies, rather than results from, sepsisrelated cardiac phenotypes, the authors employed mouse models harbouring Beclin 1 haploinsufficiency (due to the full-body knockout of one of the two Beclin 1 alleles) or a gain-of-function of Beclin 1 (due to the transgenic overexpression of the gene specifically in the heart) (10). Beclin 1 haploinsufficiency, which led to systemic attenuation of autophagy, was associated with an increased predisposition to myocardial inflammation, remodelling and dysfunction even at low LPS doses. This finding was further corroborated by reduced survival upon exposure to elevated LPS doses. In contrast, administration of Tat-Beclin 1, a cell-penetrating peptide that activates Beclin 1/autophagy, effectively reduced systemic inflammation and, more importantly, rescued cardiac function during LPS-induced sepsis both in Beclin 1 haploinsufficient and WT mice, supporting the argument for a causal role of impaired autophagy in the pathophysiology of cardiac dysfunction during sepsis. However, as both Beclin 1 haploinsufficiency and Tat-Beclin 


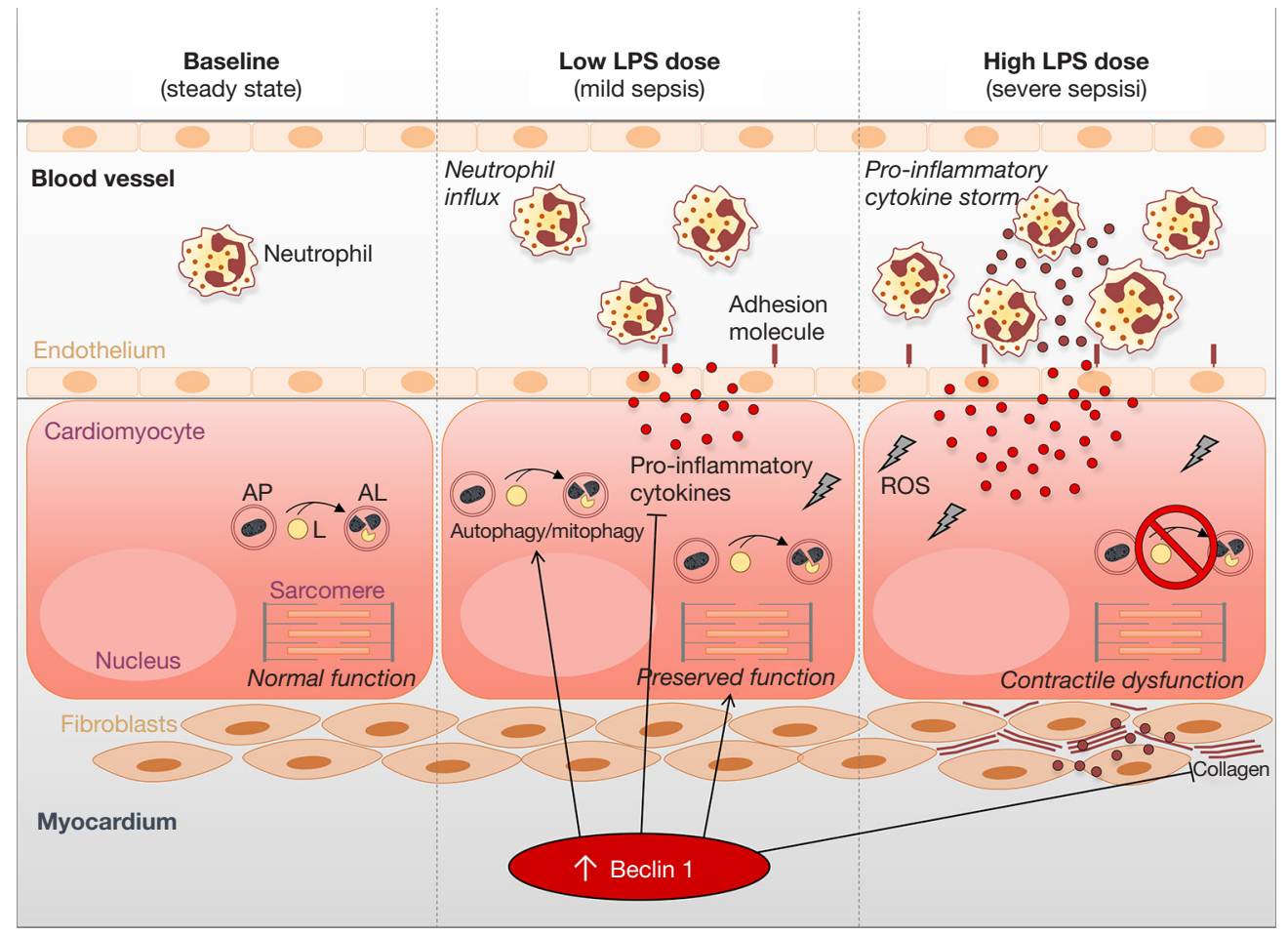

Figure 2 Beclin 1 activation counteracts LPS detrimental effects and confers cardiac resilience to sepsis. Whereas cardiac function is maintained intact during mild sepsis (induced by low LPS dose), it is impaired during severe sepsis (induced by sublethal LPS doses)—likely due to failure of persistent adaptive responses, such as autophagy. Interestingly, reinforcement of autophagy by activating Beclin 1-systemically by Tat-Beclin 1 peptide administration or locally by transgenic cardiomyocyte-specific Beclin 1 overexpression-confers cardiac resilience to high-dose LPS, as indicated by (I) preserved cardiac function, (II) reduction of pro-inflammatory cytokine production and (III) attenuation of collagen deposition. Of note, cardiomyocyte-specific Beclin 1 overexpression was sufficient to attenuate the overexpression of myocardial pro-inflammatory cytokines, including those that are not produced by the cardiomyocytes themselves. This intriguing finding could be the result of interrupted local inflammatory circuitries, whereby reduced local oxidative stress in cardiomyocytes due to activated autophagy/ mitophagy attenuates cardiomyocyte-produced cytokines (depicted as red circles). In turn, this leads to reduction of neutrophil influx and inhibition of further cytokine production by leukocytes and non-myocytes e.g., fibroblasts (depicted as brown circles). AP, autophagosome; AL, autolysosome; L, lysosome; LPS, lipopolysaccharides; ROS, reactive oxygen species.

1 peptide act at the whole-body level, it is not possible to distinguish between the anti-inflammatory effects of Beclin 1/autophagy (i.e., reduction of cytokine storm in response to LPS) versus cell-autonomous cardioprotective effects. Only the cardio-specific transgene-enforced overexpression of Beclin 1 might shed light on this dichotomy. Astonishingly enough, such a cardiomyocyte-specific Beclin 1 overexpression appeared to confer cardioprotective effects during sepsis by preserving contractility and reducing fibrosis at higher LPS doses than those endured by WT mice (10). Notably, this was coupled to an attenuated LPS-induced myocardial production of multiple proinflammatory cytokines including that of factors that most likely are not produced by cardiomyocytes themselves (such as IL-10, IL-17-alpha and IFN-gamma) (10). This suggests that the cytoprotection of cardiomyocytes by Beclin 1/ autophagy can interrupt local inflammatory circuitries put into motion by sepsis-induced cardiomyocyte stress and death. In accord with this idea, cardiomyocyte-specific Beclin 1 overexpression reduced the LPS-induced influx of neutrophils into the myocardium (Figure 2).

Enhanced autophagy in Beclin-1 transgenic hearts was also associated with improved mitochondrial function and unexpectedly high mitochondrial abundance. This perplexing observation, i.e., simultaneous mitophagy activation and increased mitochondrial mass, was attributed 
to balanced mitochondrial degradation vs. biogenesis. While Beclin 1 activates the PINK1-Parkin pathway to promote mitophagic removal of defective mitochondria, it may simultaneously stimulate mitochondrial biogenesis, at least under in vitro conditions (12). Mitochondrial biogenesis, albeit not directly measured in this study, could be further upregulated by AMPK and ULK1 (AMP-activated protein kinase and Unc-51 like autophagy activating kinase-1, respectively), both of which were found activated in septic Beclin-1 transgenic hearts. Moreover, the activation of the mitophagy receptor BNIP3 that is usually observed during sepsis was less evident upon Beclin 1 overexpression (10). This adds an additional level of complexity to Beclin 1-mediated regulation of mitochondrial homeostasis, considering that during mitochondrial damage BNIP3 is known to mediate cytoprotection (via mitophagy) or cytotoxicity (via the induction of mitochondrial transition pore opening) (13).

\section{Future perspectives and concluding remarks}

While the study substantially improves our understanding of the dynamic modulation and the cardioprotective role of autophagy and Beclin 1 in sepsis (10), some questions remain and warrant further investigation. For instance, the question as to whether Beclin 1/autophagy activation protects against sepsis-induced vascular dysfunction and hyperpermeability is still to be elucidated. In fact, pathological reduction of vascular resistance may cause cardiovascular failure and mortality even at normal or increased cardiac output, a condition known as vasoplegia. Thus, it is critical to determine the integrity of vascular endothelial barriers in sepsis upon the activation of Beclin 1-dependent autophagy. Despite the substantial protection against sublethal doses of LPS, Tat-Beclin 1 and Beclin 1 overexpression did not avoid the lethality of high LPS doses. That said, such extreme doses do not necessarily reflect the human setting where cytokines levels never reach such intensity (7). Nonetheless, it is tempting to speculate whether combining autophagy activators with other therapies, e.g., anti-inflammatory drugs, can have additive or synergistic effects to palliate this extreme, but plausibly rescuable (14), condition. Future studies will also need to test whether other xenophagy, the destruction of intracellular pathogens by a pathway resembling autophagy, may contribute to the beneficial effects of Beclin 1 by directly targeting the aetiology of sepsis, i.e., infection. In addition, together with enhanced mitochondrial function due to mitophagy, non-selective forms of autophagy may help resolve the energy crisis encountered during sepsis and that has been suggested to precipitate cardiac dysunction (15). Another aspect that warrants investigation is the mechanism(s) driving protective autophagy induction during mild sepsis. Autophagy appears to be regulated by mTORinhibitory signalling only during severe sepsis, however, this is not the case for mild sepsis, wherein autophagy induction is mediated by yet-to-be elucidated signalling pathways. Moreover, it is of utmost importance to examine whether the protective effects of Beclin 1 activation during sepsis are limited to the cardiovascular system or extend to other organs. Finally, the contribution exerted by other nonautophagic functions of Beclin 1 to health benefits awaits to be clarified.

An ever increasing body of evidence suggests that leveraging autophagy by dietary, genetic and pharmacologic interventions confers protection against undesirable cardiac alterations and functional decay associated with aging (16) and several chronic cardiac diseases (17). The study by Sun et al. (10) pleads in favour of autophagy-mediated cardioprotection against an acute cardiac injury induced by sepsis, thus, further broadening the potential therapeutic value of autophagy induction in cardiovascular medicine. In this regard, Tat-Beclin 1 peptide, which efficiently activates autophagy in vivo and protects the heart against sepsis, and other insults, like pressure overload, holds a great promise (18). Nonetheless, more efforts are needed to develop-ideally orally available-molecules that activate the autophagic activity of Beclin 1 with high specificity. It is important to note that caloric restriction mimetics that are clinically-approved (e.g., metformin) or naturallyoccurring (e.g., spermidine) (19) and have established pro-autophagic $(20,21)$, anti-inflammatory $(22,23)$, and cardioprotective effects (23-25) may provide a safe, readilyavailable, and possibly equally effective alternative for immediate testing in humans. That said, future clinical trials will reveal whether autophagy-boosting molecules will prove useful for the treatment of cardiac dysfunction caused by severe infections such as sepsis and perhaps infective endocarditis.

\section{Acknowledgements}

We acknowledge support from NAWI Graz and the BioTechMed-Graz flagship project "EPIAge".

Funding: M Abdellatif was trained within the frame of the PhD Program Molecular Medicine of the Medical 
University of Graz. S Sedej is supported by the Austrian Science Fund FWF through grants P27637-B28 and I3301-B31. S Sedej and G Kroemer are supported by the European Research Area Network on Cardiovascular Diseases (ERA-CVD, MINOTAUR). G Kroemer is supported by the Ligue contre le Cancer Comit. de Charente-Maritime (.quipe labelis.e); Agence National de la Recherche (ANR)—Projets blancs; ANR under the frame of E-Rare-2, the ERA-Net for Research on Rare Diseases; Association pour la recherche sur le cancer (ARC); Canc.rop.le Ile-de-France; Chancelerie des universit.s de Paris (Legs Poix), Fondation pour la Recherche M.dicale (FRM); a donation by Elior; the European Commission (ArtForce); the European Research Council (ERC); Fondation Carrefour; Institut National du Cancer (INCa); Inserm (HTE); Institut Universitaire de France; LeDucq Foundation; the LabEx Immuno-Oncology; the RHU Torino Lumi.re; the Seerave Foundation; the SIRIC Stratified Oncology Cell DNA Repair and Tumor Immune Elimination (SOCRATE); the SIRIC Cancer Research and Personalized Medicine (CARPEM); and the Paris Alliance of Cancer Research Institutes. F Madeo is grateful to the Austrian Science Fund FWF (Austria) for grants P23490-B20, P29262, P24381, P29203, P27893, I1000, and "SFB Lipotox" (F3012), as well as to BMWFW and the Karl-Franzens University for grant "Unkonventionelle Forschung" and grant DKplus Metabolic and Cardiovascular Diseases (W1226).

\section{Footnote}

Conflicts of Interest: The authors have no conflicts of interest to declare.

\section{References}

1. Galluzzi L, Pietrocola F, Levine B, et al. Metabolic control of autophagy. Cell 2014;159:1263-76.

2. Sica V, Galluzzi L, Bravo-San Pedro JM, et al. OrganelleSpecific Initiation of Autophagy. Mol Cell 2015;59:522-39.

3. Yin Z, Pascual C, Klionsky DJ. Autophagy: machinery and regulation. Microb Cell 2016;3:588-96.

4. Kang R, Zeh HJ, Lotze MT, et al. The Beclin 1 network regulates autophagy and apoptosis. Cell Death Differ 2011;18:571-80.

5. Qu X, Yu J, Bhagat G, et al. Promotion of tumorigenesis by heterozygous disruption of the beclin 1 autophagy gene. J Clin Invest 2003;112:1809-20.
6. Fernández ÁF, Sebti S, Wei Y, et al. Disruption of the beclin 1-BCL2 autophagy regulatory complex promotes longevity in mice. Nature 2018;558:136-40.

7. Hotchkiss RS, Karl IE. The pathophysiology and treatment of sepsis. N Engl J Med 2003;348:138-50.

8. Parrillo JE. Pathogenetic mechanisms of septic shock. N Engl J Med 1993;328:1471-7.

9. Merx MW, Weber C. Sepsis and the heart. Circulation 2007;116:793-802.

10. Sun Y, Yao X, Zhang QJ, et al. Beclin-1-Dependent Autophagy Protects the Heart During Sepsis. Circulation 2018. [Epub ahead of print].

11. Chien WS, Chen YH, Chiang PC, et al. Suppression of autophagy in rat liver at late stage of polymicrobial sepsis. Shock 2011;35:506-11.

12. Ivankovic D, Chau KY, Schapira AHV, et al. Mitochondrial and lysosomal biogenesis are activated following PINK1/parkin-mediated mitophagy. J Neurochem 2016;136:388-402.

13. Jimenez RE, Kubli DA, Gustafsson AB. Autophagy and mitophagy in the myocardium: therapeutic potential and concerns. Br J Pharmacol 2014;171:1907-16.

14. Vasques-Nóvoa F, Laundos TL, Cerqueira RJ, et al. MicroRNA-155 Amplifies Nitric Oxide/cGMP Signaling and Impairs Vascular Angiotensin II Reactivity in Septic Shock. Crit Care Med 2018;46:e945-54.

15. Drosatos K, Lymperopoulos A, Kennel PJ, et al. Pathophysiology of sepsis-related cardiac dysfunction: driven by inflammation, energy mismanagement, or both? Curr Heart Fail Rep 2015;12:130-40.

16. Abdellatif M, Sedej S, Carmona-Gutierrez D, et al. Autophagy in cardiovascular aging. Circ Res 2018;123:803-24.

17. Bravo-San Pedro JM, Kroemer G, Galluzzi L. Autophagy and Mitophagy in Cardiovascular Disease. Circ Res 2017;120:1812-24.

18. Shirakabe A, Zhai P, Ikeda Y, et al. Drp1-Dependent Mitochondrial Autophagy Plays a Protective Role Against Pressure Overload-Induced Mitochondrial Dysfunction and Heart Failure. Circulation 2016;133:1249-63.

19. Madeo F, Eisenberg T, Pietrocola F, et al. Spermidine in health and disease. Science 2018;359.

20. Eisenberg T, Knauer H, Schauer A, et al. Induction of autophagy by spermidine promotes longevity. Nat Cell Biol 2009;11:1305-14.

21. Teng ACT, Miyake T, Yokoe S, et al. Metformin increases degradation of phospholamban via autophagy in cardiomyocytes. Proc Natl Acad Sci U S A 
2015;112:7165-70.

22. Cameron AR, Morrison VL, Levin D, et al. AntiInflammatory Effects of Metformin Irrespective of Diabetes Status. Circ Res 2016;119:652-65.

23. Eisenberg T, Abdellatif M, Schroeder S, et al. Cardioprotection and lifespan extension by the natural polyamine spermidine. Nat Med 2016;22:1428-38.

Cite this article as: Abdellatif M, Sedej S, Madeo F, Kroemer G. Cardioprotective effects of autophagy induction in sepsis. Ann Transl Med 2018;6(Suppl 1):S61. doi: 10.21037/ atm.2018.10.23
24. Varjabedian L, Bourji M, Pourafkari L, et al. Cardioprotection by Metformin: Beneficial Effects Beyond Glucose Reduction. Am J Cardiovasc Drugs 2018;18:181-93.

25. Eisenberg T, Abdellatif M, Zimmermann A, et al. Dietary spermidine for lowering high blood pressure. Autophagy 2017;13:767-9. 